\title{
Chapter 1 \\ Ultrafast control of strong-field electron dynamics in solids
}

\author{
Vladislav S. Yakovlev ${ }^{1,2}$, Stanislav Yu. Kruchinin ${ }^{1}$, Tim Paasch-Colberg ${ }^{1}$, \\ Mark I. Stockman ${ }^{3}$, Ferenc Krausz ${ }^{1,2}$
}

\begin{abstract}
We review theoretical foundations and some recent progress related to the quest of controlling the motion of charge carriers with intense laser pulses and optical waveforms. The tools and techniques of attosecond science enable detailed investigations of a relatively unexplored regime of nondestructive strong-field effects. Such extremely nonlinear effects may be utilized to steer electron motion with precisely controlled optical fields and switch electric currents at a rate that is far beyond the capabilities of conventional electronics.
\end{abstract}

\subsection{Introduction}

It has long been realized that intense few-cycle laser pulses provide unique conditions for exploring extremely nonlinear phenomena in solids [1, 2], the key idea being that a sample can withstand a stronger electric field if the duration of the interaction is shortened. Ultimately, a single-cycle laser pulse provides the best conditions for studying nonperturbative strong-field effects, especially those where the properties of a sample change within a fraction of a laser cycle. The recent rapid development of the tools and techniques of attosecond science [3] not only creates new opportunities for detailed investigations of ultrafast electron dynamics in solids, but it also opens exciting opportunities for controlling electron motion in solids with unprecedented speed and accuracy. Conventional nonlinear phenomena that accompany the interaction of intense laser pulses with solids have already found a vast number of applications in spectroscopy, imaging, laser technology, transmitting and processing information [4]. It can be expected that the less conventional nonpertur-

\footnotetext{
${ }^{1}$ Max-Planck-Institut für Quantenoptik, Hans-Kopfermann-Straße 1, 85748 Garching, Germany, e-mail: vladislav. yakovlev@mpq.mpg. de

${ }^{2}$ Ludwig-Maximilians-Universität, Am Coulombwall 1, 85748 Garching, Germany

${ }^{3}$ Center for Nano-Optics (CeNO) and Department of Physics and Astronomy, Georgia State University, Atlanta, Georgia 30340, USA
} 
bative nonlinearities may also find important applications [5, 6]. The main purpose of this chapter is to review theoretical foundations and some recent progress in this field.

Most of the relevant physical phenomena are well known, such as interband tunneling, Franz-Keldysh effect, Bloch oscillations, and Wannier-Stark localization. However, new experiments put these phenomena in a new context, which often leads to nontrivial observations, such as the generation of nonperturbative high-order harmonics in a solid due to Bragg-like scattering at the edges of the Brillouin zone $[7,8]$, a nearly instantaneous change in extreme-ultraviolet absorptivity and nearinfrared reflectivity of a dielectric in the presence of a laser field as strong as several volts per ångström [9], or the induction of electric current in an unbiased dielectric by similarly intense laser pulses [10]. Several decades of research on strong-field phenomena in solids and mesoscopic structures provide a solid ground for developing new theoretical models adapted for new experimental conditions. At the same time, a description of extremely nonlinear processes that unfold during just a few femtoseconds requires approximations that may be different from those established for longer and less intense laser pulses. Both $a b$ initio calculations that attempt to capture all the complexity of many-electron dynamics and "toy models" designed to deepen our understanding of basic phenomena are going to play an important role in extending our ability to control the optical and electric properties of solids with controlled light fields.

The key parameters that determine the regime of strong-field light-matter interaction with dielectrics and semiconductors are the Keldysh parameter

$$
\gamma_{\mathrm{K}}=\frac{\omega_{\mathrm{L}} \sqrt{m E_{g}}}{e F_{\mathrm{L}}}
$$

the Bloch frequency

$$
\omega_{\mathrm{B}}=\frac{e F_{\mathrm{L}} a}{\hbar},
$$

and the Rabi frequency

$$
\Omega_{\mathrm{R}}=\frac{d_{\mathrm{cV}} F_{\mathrm{L}}}{\hbar} .
$$

Here, $F_{\mathrm{L}}$ is the amplitude of a linearly polarized electric field oscillating at an angular frequency $\omega_{\mathrm{L}}, e>0$ is the absolute value of the electron charge, $m$ is the reduced mass of an electron and a hole $\left(m^{-1}=m_{\mathrm{e}}^{-1}+m_{\mathrm{h}}^{-1}\right), a$ is the lattice period in the polarization direction of the electric field, and $d_{\mathrm{cv}}$ is the dipole matrix element responsible for transitions between valence- and conduction-band states. Conditions that have to be fulfilled for perturbation theory to be applicable include $\gamma_{\mathrm{K}} \gg 1$, $\omega_{\mathrm{B}} \ll \omega_{\mathrm{L}}, \Omega_{\mathrm{R}} \ll \omega_{\mathrm{L}}$, and $\hbar \Omega_{\mathrm{R}} \ll E_{\mathrm{g}}$. Correspondingly, the main physical effects that make the interaction nonperturbative are interband tunneling $\left(\gamma_{\mathrm{K}} \lesssim 1\right)$, Bloch oscillations $\left(\omega_{\mathrm{B}} \geq \pi \omega_{\mathrm{L}}\right)$, Rabi flopping ( $\left.\hbar \Omega_{\mathrm{R}} \gtrsim E_{\mathrm{g}}\right)$, and carrier-wave Rabi flopping $\left(\Omega_{\mathrm{R}} \gtrsim \omega_{\mathrm{L}}\right)$, the last two effects being particularly important for resonant excitations $\left(\hbar \omega_{\mathrm{L}} \approx E_{\mathrm{g}}\right)$. For a band gap of several electronvolts and a laser frequency in the 
near-infrared spectral range $\left(\lambda_{\mathrm{L}}=2 \pi c / \omega_{\mathrm{L}} \sim 1 \mu \mathrm{m}\right)$, these effects become essential for $F_{\mathrm{L}} \gtrsim 1 \mathrm{~V} / \AA$, which corresponds to a laser intensity of $I_{\mathrm{L}} \gtrsim 10^{13} \mathrm{~W} / \mathrm{cm}^{2}$ [11].

\subsection{Main theoretical concepts}

For a theoretical description of phenomena that take place on a few-femtosecond time scale, it is common (although not necessarily correct) to neglect dephasing. In this case, the time-dependent Schrödinger equation (TDSE)

$$
\mathrm{i} \hbar \partial_{t} \psi(t)=\hat{H}(t) \psi(t)
$$

fully describes nonrelativistic electron dynamics. In situations where phase and energy relaxation processes are important, one has to use the more general formalisms of density matrices or nonequilibrium Green's functions, which are beyond the scope of this chapter. In the following, we review some theoretical concepts developed for the case of a single charged particle moving in a periodic potential. Furthermore, since the wavelength of visible or infrared light is much larger than the size of a unit cell, and electron velocities are much smaller than the speed of light, we can use the dipole approximation, which neglects the spatial dependence of the laser field while solving the TDSE: $\mathbf{F}_{\mathrm{L}}=\mathbf{F}_{\mathrm{L}}(t)$.

The first step to solve the TDSE is to choose a gauge and a basis. The exact solution does not depend on this choice, but the chosen gauge and basis dictate approximations that one may wish to make, and they influence the physical interpretation of results. In the dipole approximation, the two main options are the velocity and length gauges, which, in the following, will be abbreviated as "VG" and "LG", respectively. These two gauges are related to each other by the following unitary transformation of the respective wave functions $\psi_{\mathrm{VG}}$ and $\psi_{\mathrm{LG}}$ :

$$
\psi_{\mathrm{VG}}(t)=\exp \left[-\frac{\mathrm{i}}{\hbar} e \mathbf{A}_{\mathrm{L}}(t) \mathbf{r}\right] \psi_{\mathrm{LG}}(t)
$$

where

$$
\mathbf{A}_{\mathrm{L}}(t)=-\int^{t} \mathbf{F}_{\mathrm{L}}\left(t^{\prime}\right) \mathrm{d} t^{\prime}
$$

is the vector potential of the laser field.

The Hamilton operators in these two gauges take the following forms:

$$
\begin{aligned}
& \hat{H}_{\mathrm{VG}}=\frac{\left[\hat{\mathbf{p}}+e \mathbf{A}_{\mathrm{L}}(t)\right]^{2}}{2 m}+U(\mathbf{r}), \\
& \hat{H}_{\mathrm{LG}}=\frac{\hat{\mathbf{p}}^{2}}{2 m}+U(\mathbf{r})+e \mathbf{F}_{\mathrm{L}}(t) \mathbf{r} .
\end{aligned}
$$

A big advantage of the velocity gauge for numerical simulations is that a homogeneous external field does not destroy the spatial periodicity of the Hamiltonian, 
so that the Bloch theorem applies even in the presence of the field. At the same time, transformation (1.5) can be interpreted as a transition to a moving coordinate system, in which electrons acquire an additional momentum $-e \mathbf{A}_{\mathrm{L}}(t)$. In order to accurately account for such superficial dynamics in a time-independent basis, a sufficient number of basis states and exact transition matrix elements are required. Calculations in the velocity gauge are particularly problematic in the limit of a static field $\left(\omega_{\mathrm{L}} \rightarrow 0\right)$.

These problems are circumvented in the length gauge. The price for this is the fact that the term $e \mathbf{F}_{\mathrm{L}}(t) \mathbf{r}$, which is responsible for the interaction with an external field, destroys the spatial periodicity of the Hamiltonian. Also, when periodic boundary conditions are applied in the length gauge, the interaction potential becomes discontinuous at the boundaries of the unit cell. Nevertheless, once these difficulties are addressed, the length gauge becomes an appropriate choice for numerical simulations [12-16].

When the field of a laser pulse is nonresonant $\left(\hbar \omega_{\mathrm{L}} \ll E_{\mathrm{g}}\right)$ and strong, it is frequently convenient to use a time-dependent basis of quantum states that adiabatically "adapt" themselves to the external field. In the length gauge, such an adiabatic basis is given by Wannier-Stark states. In the velocity gauge, this role is played by accelerated Bloch states, also known as Houston functions [17]. The following two subsections summarize the most important properties of these states.

\subsubsection{Wannier-Stark resonances}

For simplicity, let us consider a one-dimensional problem. For a constant external field parallel to the $z$-axis, the stationary Schrödinger equation reads

$$
\hat{H}_{\mathrm{LG}} \psi \equiv\left(\frac{\hat{p}^{2}}{2 m}+U(z)+e F_{\mathrm{L}} z\right) \psi=E \psi .
$$

The potential here is periodic: $U(z+a)=U(z)$ with a lattice period $a$. Leaving the question of the existence of such eigenstates aside, one can ask which properties the solutions of (1.9) possess if they exist. From the periodicity of the potential, we immediately conclude that if $\psi(z)$ is an eigenstate with an energy $E$, then $\psi(z-a)$ is also an eigenstate with the energy $E+e F_{\mathrm{L}} a$. The additional term $e F_{\mathrm{L}} a$ is the energy required to move an electron against the laser field by one lattice period. This suggests that the eigenstates $\psi(z)$ should be localized functions. They are referred to as Wannier-Stark states.

Wannier found approximate solutions to (1.9) by defining an auxiliary problem

$$
\left(\frac{\hat{p}^{2}}{2 m}+U(z)+e F_{\mathrm{L}}\left[z+\mathrm{i} \frac{\partial}{\partial k}\right]\right) b_{i}(z, k)=E_{i}(k) b_{i}(z, k),
$$

where $i$ is a band index, $k$ is the crystal momentum, and $E_{i}(k)$ is the energy of the unperturbed Bloch state. Wannier showed [18] that the solutions of this problem, 
known as Wannier-Bloch states, decouple different bands. Specifically, knowing $b_{i}(z, k)$ that satisfies the periodic boundary condition in reciprocal space $b_{i}(z, k+$ $2 \pi / a)=b_{i}(z, k)$, an approximate solution of the time-dependent Schrödinger equation with the Hamiltonian $H_{\mathrm{LG}}$ can be written as

$$
\psi_{i}(z, t)=b_{i}\left(z, k_{0}-\frac{e F_{\mathrm{L}}}{\hbar} t\right) \exp \left[-\frac{\mathrm{i}}{\hbar} \int_{t_{0}}^{t} E_{i}\left(k_{0}-\frac{e F_{\mathrm{L}}}{\hbar} t^{\prime}\right) \mathrm{d} t^{\prime}\right],
$$

where the approximation consists in restricting the electron motion to a single band, that is, neglecting interband transitions. Obviously, $\left|\psi_{i}(z, t)\right|^{2}$ is a periodic function of time, the period being equal to the period of Bloch oscillations: $T_{\mathrm{B}}=2 \pi / \omega_{\mathrm{B}}=$ $2 \pi \hbar /\left(e F_{\mathrm{L}} a\right)$. The Wannier-Stark states for a bulk crystal $\psi_{i, \ell}^{\mathrm{WS}}(z)$ are defined via the expansion

$$
\psi_{i}(z, t)=\sum_{\ell} \psi_{i, \ell}^{\mathrm{WS}}(z) \exp \left[-\frac{\mathrm{i}}{\hbar} E_{i, \ell}^{\mathrm{WS}} t\right] .
$$

A state $\psi_{i, \ell}^{\mathrm{WS}}(z)$ is localized at a lattice site $\ell$. Explicit expressions for the WannierStark states and their energies are

$$
\begin{gathered}
\psi_{i, \ell}^{\mathrm{WS}}(z)=\frac{a}{2 \pi} \int_{-\pi / a}^{\pi / a} \mathrm{~d} k b_{i}(z, k) \mathrm{e}^{-\mathrm{i} \ell a k}, \\
E_{i, \ell}^{\mathrm{WS}}=\bar{E}_{i}+\ell e a F_{\mathrm{L}}
\end{gathered}
$$

where $\bar{E}_{i}$ is the mean energy of band $i$ :

$$
\bar{E}_{i}=\frac{a}{2 \pi} \int_{-\pi / a}^{\pi / a} \mathrm{~d} k E_{i}(k)
$$

The energies $E_{i, \ell}^{\mathrm{WS}}$ form the so-called "Wannier-Stark ladder"-plotted against $F_{\mathrm{L}}$, they are a set of straight lines, where the slope of each line $\mathrm{d} E_{i, \ell}^{\mathrm{WS}} / \mathrm{d} F_{\mathrm{L}}=\ell e a$ is determined by the lattice index $\ell$.

The localization length of a Wannier-Stark state is given by

$$
L_{i}^{\mathrm{WS}}=\frac{\Delta_{i}}{e\left|F_{\mathrm{L}}\right|}
$$

where $\Delta_{i}$ is the energy interval covered by band $i$.

Our introduction to the Wannier-Stark states has so far followed the one given by Wannier [19]. The existence of Wannier-Stark states had been a subject of numerous disputes for three decades until they were experimentally observed in superlattices [20]. The core of these disputes was the question whether the Wannier-Stark states retain their physical significance when interband transitions are accounted for [21-23]. According to modern treatments [24-27], Wannier-Stark states should be viewed as resonances (metastable states) with lifetimes $\tau_{i}^{\mathrm{WS}}=1 / \Gamma_{i}$, and (1.14) should be generalized as 


$$
E_{i, \ell}^{\mathrm{WS}}=\bar{E}_{i}+\left(\ell-\frac{\gamma_{i}^{\mathrm{Zak}}}{2 \pi}\right) e a F_{\mathrm{L}}-\mathrm{i} \hbar \frac{\Gamma_{i}}{2}
$$

Here, $\Gamma_{i}$ is the decay rate due to interband transitions, and

$$
\gamma_{i}^{\mathrm{Zak}}=\oint_{\mathrm{BZ}} \mathrm{d} \mathbf{k} \cdot \boldsymbol{\xi}_{i i}(\mathbf{k})
$$

is called Zak's phase [25, 28], where the integral is taken over a smooth closed path across the entire Brillouin zone, and $\boldsymbol{\xi}_{i i}(\mathbf{k})$ is the Berry connection or geometric vector potential [25-27]. An explicit expression for $\boldsymbol{\xi}_{i j}(\mathbf{k})$ is given by (1.26) in the next section. Zak's phase plays an important role in the "modern theory of polarization" [29]; it is equal to either 0 or $\pi$ for crystals that possess inversion symmetry, and it can assume any value for other crystals. Direct measurements of Zak's phase were performed for cold atoms in optical lattices [30].

Much of the mathematical complexity related to Wannier-Stark states is avoided in finite systems, where the electron motion is restricted. In this case, it is common to refer to the exact length-gauge eigenstates of $\hat{H}_{\mathrm{LG}}$ as Wannier-Stark states (without neglecting interband transitions by evaluating Wannier-Bloch states). These states have properties similar to those of the states introduced by Wannier. One of the most important differences is that the exact eigenstates of a Hamiltonian in one spatial dimension may not be degenerate. While the states defined by (1.14) are strictly linear functions of $F_{\mathrm{L}}$, and, for a certain value of $F_{\mathrm{L}}$, some of these energies $E_{i_{1}, \ell_{1}}^{\mathrm{WS}}$ and $E_{i_{2}, \ell_{2}}^{\mathrm{WS}}$ may be equal to each other, the corresponding exact energies of a confined quantum system will have avoided crossings (anticrossings). An example of such a Wannier-Stark ladder is shown in Fig. 1.5.

\subsubsection{Accelerated Bloch states}

In section 1.2.1, we saw that the instantaneous eigenstates of the length-gauge Hamiltonian serve as a convenient basis for developing approximate solutions to the TDSE. In situations where the difficulties related to the length gauge outweigh its advantages, the velocity gauge may be a better choice for either numerical or analytical approximations, and the instantaneous eigenstates of the velocity-gauge Hamiltonian (1.7) may provide a more useful time-dependent basis.

Let $\phi_{i, \mathbf{k}}$ be a Bloch state with a band index $i$ and a crystal momentum $\mathbf{k}$ :

$$
\left(\frac{\hat{\mathbf{p}}^{2}}{2 m}+U(\mathbf{r})\right) \phi_{i, \mathbf{k}}=E_{i}(\mathbf{k}) \phi_{i, \mathbf{k}} .
$$

In the coordinate representation, $\phi_{i, \mathbf{k}}(\mathbf{r})$ is a product of a plane wave and a latticeperiodic envelope function:

$$
\phi_{i, \mathbf{k}}(\mathbf{r})=\mathrm{e}^{\mathrm{i} \mathbf{k r}} u_{i, \mathbf{k}}(\mathbf{r})
$$


where $u_{i, \mathbf{k}}(\mathbf{r}+\mathbf{R})=u_{i, \mathbf{k}}(\mathbf{r})$ for all $\mathbf{R}$ from the Bravais lattice. Let us now consider the instantaneous eigenstates of $\hat{H}_{\mathrm{VG}}$ in the presence of a homogeneous external field:

$$
\left(\frac{\left[\hat{\mathbf{p}}+e \mathbf{A}_{\mathrm{L}}(t)\right]^{2}}{2 m}+U(\mathbf{r})\right) \varphi(t)=\widetilde{E}(t) \varphi(t) .
$$

Since the Hamiltonian is periodic in space, the Bloch theorem is applicable. Equation (1.20) has the same form as (1.18), the momentum operator being substituted with $\hat{\mathbf{p}}+e \mathbf{A}_{\mathrm{L}}(t)$. The requirement that the solutions of (1.20) satisfy the Born-von Kármán boundary conditions yields [31]

$$
\begin{aligned}
\varphi_{i, \mathbf{k}_{0}}(\mathbf{r}, t) & =\exp \left[-\frac{\mathrm{i}}{\hbar} e \mathbf{A}_{\mathrm{L}}(t) \mathbf{r}\right] \phi_{i, \mathbf{k}(t)}(\mathbf{r}), \\
\widetilde{E}_{i, \mathbf{k}_{0}}(t) & =E_{i}(\mathbf{k}(t)),
\end{aligned}
$$

where the time-dependent crystal momentum

$$
\mathbf{k}(t)=\mathbf{k}_{0}+\frac{e}{\hbar} \mathbf{A}_{\mathbf{L}}(t)
$$

satisfies the acceleration theorem: $\hbar \mathrm{d} \mathbf{k} / \mathrm{d} t=-e \mathbf{F}_{\mathrm{L}}(t)$. Here, $\mathbf{k}_{0}$ is the initial crystal momentum, which the electron possessed prior to the interaction with the laser pulse.

The states $\varphi_{i, \mathbf{k}_{0}}(\mathbf{r}, t)$ are called accelerated Bloch states or Houston functions. We note that there is a close analogy between these functions and Volkov solutions of the TDSE [32], which are frequently used for describing the strong-field ionization of atoms and molecules [33].

To use Houston functions as a basis for solving the TDSE, it is convenient to make the ansatz

$$
\psi_{\mathbf{k}_{0}}(\mathbf{r}, t)=\sum_{i} \alpha_{i, \mathbf{k}_{0}}(t) \exp \left[-\frac{\mathrm{i}}{\hbar} \int_{t_{0}}^{t} \mathrm{~d} t^{\prime} E_{i}\left(\mathbf{k}\left(t^{\prime}\right)\right)\right] \varphi_{i, \mathbf{k}_{0}}(\mathbf{r}, t),
$$

which leads to the following system of differential equations [31, 34]:

$$
\frac{\mathrm{d}}{\mathrm{d} t} \alpha_{i, \mathbf{k}_{0}}(t)=\frac{e}{\mathrm{i} \hbar} \sum_{j} \alpha_{j, \mathbf{k}_{0}}(t) \mathbf{F}_{\mathrm{L}}(t) \cdot \boldsymbol{\xi}_{i j}(\mathbf{k}(t)) \exp \left[\frac{\mathrm{i}}{\hbar} \int_{t_{0}}^{t} \mathrm{~d} t^{\prime} \Delta E_{i j}\left(\mathbf{k}\left(t^{\prime}\right)\right)\right] .
$$

Here, $\Delta E_{i j}(\mathbf{k})=E_{i}(\mathbf{k})-E_{j}(\mathbf{k})$, and the matrix elements

$$
\boldsymbol{\xi}_{i j}(\mathbf{k}) \equiv\left\langle i, \mathbf{k}\left|\mathrm{i} \nabla_{\mathbf{k}}\right| j, \mathbf{k}\right\rangle_{\text {cell }}=\frac{\mathrm{i}}{\Omega} \int_{\Omega} \mathrm{d}^{3} r u_{i, \mathbf{k}}^{*}(\mathbf{r}) \nabla_{\mathbf{k}} u_{j, \mathbf{k}}(\mathbf{r})
$$

describe the optical transitions between bands, where the integration is performed over the volume $\Omega$ of a unit cell.

The relation between $\boldsymbol{\xi}_{i j}(\mathbf{k})$ and the momentum matrix elements 


$$
\mathbf{p}_{i j}(\mathbf{k}) \equiv\langle i, \mathbf{k}|\hat{\mathbf{p}}| j, \mathbf{k}\rangle_{\text {cell }}=-\frac{\mathrm{i} \hbar}{\Omega} \int_{\Omega} \mathrm{d}^{3} r u_{i, \mathbf{k}}^{*}(\mathbf{r}) \nabla_{\mathbf{r}} u_{j, \mathbf{k}}(\mathbf{r})
$$

is given by

$$
\boldsymbol{\xi}_{i \neq j}(\mathbf{k})=\frac{\mathrm{i} \hbar \mathbf{p}_{i j}(\mathbf{k})}{m_{0} \Delta E_{i j}(\mathbf{k})}
$$

where $m_{0}$ is the electron rest mass. This expression can only be applied as long as $\Delta E_{i j}(\mathbf{k})$ in the denominator is not equal to zero. The case of degenerate bands presents additional mathematical challenges [27,35,36]; in particular, the transition matrix elements $\boldsymbol{\xi}_{i j}(\mathbf{k})$ are singular at degeneracies [36].

For reference, we also give the relation between $\boldsymbol{\xi}_{i j}(\mathbf{k})$ and the matrix elements of the position operator between the Bloch functions [15, 37, 38]:

$$
\left\langle i, \mathbf{k}^{\prime}|\hat{\mathbf{r}}| j, \mathbf{k}\right\rangle_{\infty}=\int_{\mathbb{R}^{3}} \mathrm{~d}^{3} r \phi_{i, \mathbf{k}^{\prime}}^{*}(\mathbf{r}) \mathbf{r} \phi_{j, \mathbf{k}}(\mathbf{r})=\left[\mathrm{i} \delta_{i j} \nabla_{\mathbf{k}}+\boldsymbol{\xi}_{i j}(\mathbf{k})\right] \delta\left(\mathbf{k}-\mathbf{k}^{\prime}\right) .
$$

In the case $\alpha_{i, \mathbf{k}_{0}}\left(t_{0}\right)=\delta_{i j}$, where an electron is in band $j$ before the external field is turned on, a simple approximate solution to (1.25) is [31]

$$
\alpha_{i \neq j, \mathbf{k}_{0}}(t) \approx \frac{e}{\mathrm{i} \hbar} \int_{t_{0}}^{t} \mathrm{~d} t^{\prime} \mathbf{F}_{\mathrm{L}}\left(t^{\prime}\right) \cdot \boldsymbol{\xi}_{i j}\left(\mathbf{k}\left(t^{\prime}\right)\right) \exp \left[\frac{\mathrm{i}}{\hbar} \int_{t_{0}}^{t^{\prime}} \mathrm{d} t^{\prime \prime} \Delta E_{i j}\left(\mathbf{k}\left(t^{\prime \prime}\right)\right)\right],
$$

provided that the excitation probabilities are small $\left(\left|\alpha_{i, \mathbf{k}_{0}}\right|^{2} \ll 1\right.$ for $\left.i \neq j\right)$.

This equation is a good starting point for numerous analytical approximations. In the case of a constant external field $\left(\mathbf{F}_{\mathrm{L}}=\right.$ const $)$, it is convenient to rewrite the right-hand side as an integral over the crystal momentum $\mathbf{k}$. Alternatively, the integral over time can be approximately evaluated using the saddle-point method, which is especially useful in the case of a monochromatic external field. Such approximations can be used to obtain analytical expressions for the rate of strong-field-induced transitions between valence and conduction bands of a dielectric or a semiconductor. These transitions belong to the most important strong-field effects in solids, and they are discussed in the next subsection.

\subsubsection{Nonresonant interband transitions}

In 1928, Zener [39] used semiclassical arguments to show that a constant external field $F$ makes valence-band electrons of a dielectric tunnel to the conduction band at a rate (per unit volume)

$$
\Gamma_{\text {Zener }}=\frac{e|F| a}{2 \pi \hbar} \exp \left[-\frac{\pi}{2} \frac{m^{1 / 2} E_{\mathrm{g}}^{3 / 2}}{e \hbar|F|}\right] .
$$

The prefactor of the exponential function was found to be rather sensitive to a chosen method of approximation, but all such methods yield the same argument of 
the exponential function. For example, Kane derived [40] the tunneling rate to be equal to

$$
\Gamma_{\text {Kane }}=\frac{e^{2} F^{2} m^{1 / 2}}{18 \pi \hbar^{2} E_{\mathrm{g}}^{1 / 2}} \exp \left[-\frac{\pi}{2} \frac{m^{1 / 2} E_{\mathrm{g}}^{3 / 2}}{e \hbar|F|}\right],
$$

while Keldysh obtained [41]

$$
\Gamma_{\text {Keldysh }}=\frac{2|e F|^{5 / 2} m^{1 / 4}}{9 \pi^{2} \hbar^{3 / 2} E_{\mathrm{g}}^{5 / 4}} \exp \left[-\frac{\pi}{2} \frac{m^{1 / 2} E_{\mathrm{g}}^{3 / 2}}{e \hbar|F|}\right] .
$$

It is common to state that such expressions for the tunneling rate have an "exponential accuracy" [42].

If the external field is not constant but oscillating at a constant frequency, interband transitions may also occur as a result of absorbing a number of photons sufficient to overcome the band gap. It must be emphasized that there is no sharp distinction between nonresonant interband tunneling and multiphoton transitions. These are two asymptotic cases of interband excitations, which are distinguished by the Keldysh parameter (1.1): $\gamma_{\mathrm{K}} \gg 1$ for multiphoton excitations and $\gamma_{\mathrm{K}} \ll 1$ for tunneling. Equation (1.31) is valid in the latter case, where the laser field is strong and its frequency is small. In the intermediate regime $\left(\gamma_{\mathrm{K}} \sim 1\right)$, it is impossible to unambiguously distinguish between contributions from multiphoton absorption and tunneling. This is reminiscent of the situation in atomic physics where the electron motion under the potential barrier (tunneling) is known to be important even in the multiphoton regime [43]. Keldysh also derived a more general expression for the transition rate averaged over a laser cycle, which is applicable in all the three regimes [41]:

$$
\begin{aligned}
& \Gamma_{\mathrm{GKF}}=\frac{2 \omega_{\mathrm{L}}}{9 \pi}\left[\frac{m \omega_{\mathrm{L}}}{\hbar \beta}\right]^{3 / 2} Q\left(\gamma_{\mathrm{K}}, \widetilde{N}\right) \exp \left[-\pi\lfloor\widetilde{N}+1\rfloor \frac{K(\beta)-E(\beta)}{E(\alpha)}\right], \\
& Q\left(\gamma_{\mathrm{K}}, \widetilde{N}\right)=\left[\frac{\pi}{2 K(\alpha)}\right]^{1 / 2} \sum_{n=0}^{\infty} \exp \left[-\frac{\pi n[K(\beta)-E(\beta)]}{E(\alpha)}\right] \\
& \times \Phi\left\{\left[\frac{\pi^{2}(2\lfloor\widetilde{N}+1\rfloor-2 \widetilde{N}+N)}{2 K(\alpha) E(\alpha)}\right]^{1 / 2}\right\} \\
& \alpha=\left(1+\gamma_{\mathrm{K}}^{2}\right)^{-1 / 2}, \quad \beta=\gamma_{\mathrm{K}} \alpha, \quad \widetilde{N}=\frac{\widetilde{E}_{\mathrm{g}}}{\hbar \omega_{\mathrm{L}}}, \quad \widetilde{E}_{\mathrm{g}}=\frac{2 E(\alpha)}{\pi \beta} E_{\mathrm{g}}, \quad N=\frac{E_{\mathrm{g}}}{\hbar \omega_{\mathrm{L}}} .
\end{aligned}
$$

Here, $\widetilde{E}_{\mathrm{g}}$ is an effective ionization potential, the functions $K(z)$ and $E(z)$ are the complete elliptic integrals of first and second kind, $\Phi(z)$ is the Dawson function, and $\lfloor x\rfloor$ denotes the integer part of $x$. We refer to (1.32) as the general Keldysh formula $(\mathrm{GKF})$. 


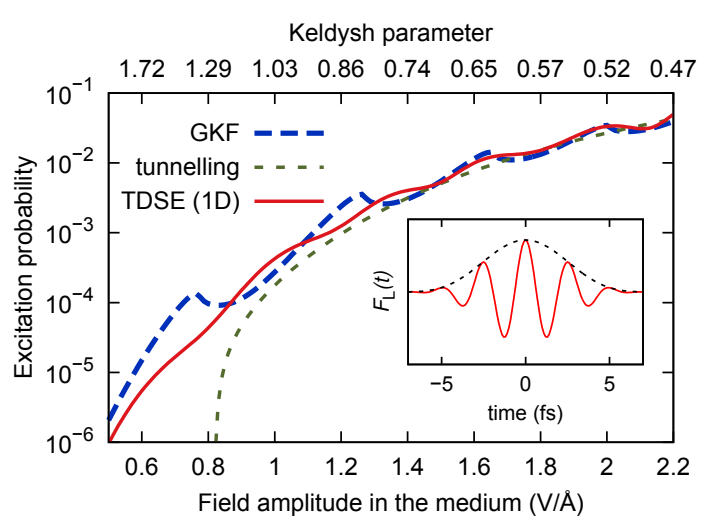

Fig. 1.1 The probability that a 4-fs laser pulse excites a valence-band electron of $\mathrm{SiO}_{2}$ to one of the conduction bands. The laser pulse, shown in the inset, has a central wavelength of $\lambda_{\mathrm{L}}=800 \mathrm{~nm}$. The dashed curves show probabilities evaluated by integrating analytical excitation rates: $1-\exp \left[-\int \Gamma(t) \mathrm{d} t\right]$. For the curve marked as 'GKF', the general Keldysh formula (1.32) was used as a cycle-averaged excitation rate; evaluating $\Gamma_{\mathrm{GKF}}(t)$ we substituted $F_{\mathrm{L}}$ with the envelope of the laser pulse (dashed line in the inset). For the curve marked as 'tunneling', we used the quasistatic approximation in the tunneling limit $\Gamma(t)=\Gamma_{\text {Keldysh }}(t)$ by substituting $F_{\mathrm{L}}$ with the electric field $F_{\mathrm{L}}(t)$ in (1.31). The solid curve is a numerical result obtained by solving the TDSE in a 1D model [44]. The transition rates (1.31) and (1.32) were multiplied with constant factors to match the TDSE result for $\gamma_{\mathrm{K}} \lesssim 1$.

Are these formulas, which were obtained decades ago for a monochromatic external field, still useful in the case of few-cycle laser pulses? To address this question, we present, in Fig. 1.1, the outcomes of a simulation where a 4-fs 800-nm laser pulse interacts with a one-dimensional model medium that has properties resembling those of $\alpha$-quartz [44]: a band gap of $E_{\mathrm{g}}=9 \mathrm{eV}$, a lattice period of $5 \AA$, and a reduced mass of $m=0.38 m_{0}$. To compare the numerically evaluated excitation probabilities (solid curve) with the GKF predictions, we evaluated the excitation rate $\Gamma_{\mathrm{GKF}}(t)$ using the real-valued pulse envelope in the place of the electric field $F_{\mathrm{L}}$, which enters (1.32) via $\gamma_{\mathrm{K}}$. From $\Gamma_{\mathrm{GKF}}(t)$, we estimated the excitation probability as $p_{\mathrm{GKF}} \propto 1-\exp \left[-\int \Gamma_{\mathrm{GKF}}(t) \mathrm{d} t\right]$ (thick dashed curve). The overall agreement is surprisingly good, given the fact that the laser pulse is shorter than two optical periods. Both the numerical and GKF results exhibit an oscillatory behavior, which appears due to closing and opening of multiphoton channels. It is analogous to the channel closing phenomenon in atomic physics [45-47]. The thin dashed curve in Fig. 1.1 represents the excitation probability evaluated with (1.31). Since $\Gamma_{\text {Keldysh }}$ is the tunneling rate for a constant field, we used $F_{\mathrm{L}}(t)$ (rather than the pulse envelope) to evaluate the excitation probability by the laser pulse. This procedure is known as the quasistatic approximation [48]. In this example, the tunneling formula (1.31) is inaccurate for $F_{\mathrm{L}} \lesssim 1.2 \mathrm{~V} / \AA$, which corresponds to $\gamma_{\mathrm{K}} \gtrsim 0.9$.

The pioneering work by Keldysh was followed by numerous investigations. A few that we would like to point out here are analytical results obtained using the adiabatic approach, where parity selection rules were accounted for [34, 49], derivation 
of expressions for arbitrary $\mathrm{N}$-photon transition probabilities [50], and development of the Keldysh-like theory for cosine-shaped bands [51].

\subsection{Strong-field-driven electron dynamics in crystals}

\subsubsection{A numerical example}

A strong electric field drives interband transitions, it accelerates charge carriers, and it can also cause transient changes in the optical properties of a medium without necessarily exciting electrons to conduction bands. Combined with controlled optical fields, these three classes of physical phenomena enable the manipulation of the electric and optical properties of a medium over time intervals much shorter than a period of optical oscillations. The main examples of such controlled fields are laser pulses with the stabilized carrier-envelope phase (CEP) and optical waveforms [52].

We begin this section by illustrating such effects in a simulation where the TDSE was solved in one spatial dimension for a periodic potential. In Fig. 1.2, we show $\left|\alpha_{i, k(t)}(t)\right|^{2}$ obtained by solving the Houston-basis equations (1.25) and representing the conduction-band populations in the extended-zone scheme, where the range of crystal momenta covered by the $n$-th conduction band $(n \geq 1)$ is $n-1 \leq|k| / k_{\max } \leq n$ with $k_{\max }=\pi / a$. According to the acceleration theorem, the ballistic motion of an electron wave packet is described by $\hbar \mathrm{d}\langle k\rangle / \mathrm{d} t=-e F_{\mathrm{L}}(t)$, where $\langle k\rangle$ is the mean crystal momentum. A continuous change of $\langle k\rangle$ at the borders $|k| / k_{\max }=n$ corresponds to transitions between different conduction bands. Such interband transitions are closely related to Landau-Zener transitions, as they occur at crystal momenta where the energy gap between two adjacent bands is particularly small. If a charge carrier remains within its current band as it crosses a Brillouin zone edge, its crystal momentum changes abruptly. This can be interpreted as a Bragg-like reflection of an electron wave off the crystal lattice. Fig. 1.2 shows that both Bragg reflections and interband transitions play an important role when the electron motion is driven by a near-infrared field.

In Fig. 1.2(a), the electron wave packet is initially placed in the lowest conduction band of the 1D model of a solid, and even though the laser pulse with $\lambda_{\mathrm{L}}=1.6 \mu \mathrm{m}$ is strong enough to accelerate electrons out of the first Brillouin zone, its amplitude is insufficient to induce transitions between conduction and valence bands. The figure illustrates the importance of transitions between different conduction bands, and shows that they predominantly occur at $k=\pi n / a, n \in \mathbb{Z}$.

For the simulations presented in Fig. 1.2(b), all electrons were initially placed in the valence bands. The laser pulse had the same peak value of the vector potential as in Fig. 1.2(a), but it had a shorter wavelength $\lambda_{\mathrm{L}}=800 \mathrm{~nm}$, so that the electric field of the pulse was twice as strong, and it was strong enough to excite electrons from the uppermost valence band. One can see that the population of conduction bands does not constantly increase with time, as one would expect from rate models 
(a)

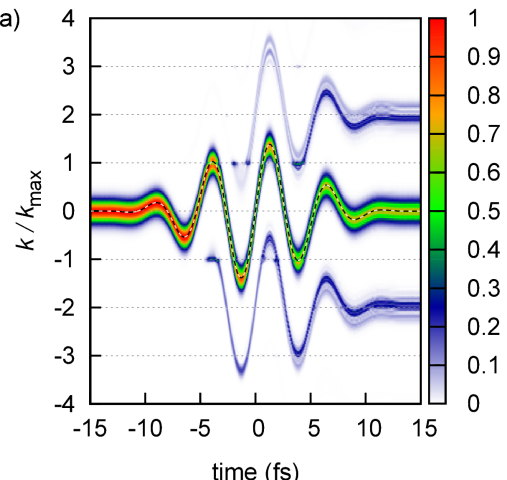

(b)

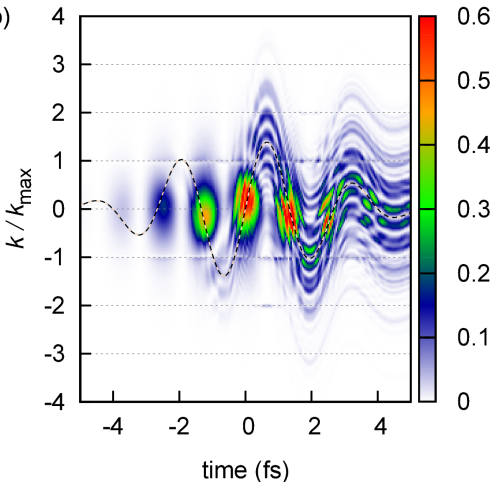

Fig. 1.2 The time-dependent population distribution of conduction-band electrons in the presence of a few-cycle laser pulse. These plots were obtained by solving (1.25) for a 1D model of $\mathrm{SiO}_{2}$ with the same lattice potential as that used in [44]. The normalized conduction-band population $(\max n(t, k)=1)$ is plotted against time and crystal momentum $k$ in the extendedzone scheme, where the range of crystal momenta covered by the $n$-th lowest conduction band is $n-1 \leq|k| / k_{\max } \leq n$ with $k_{\max }=\pi / a$. The dashed curves show $A_{\mathrm{L}} / k_{\max }$. (a) An electron wave packet is initially placed in the lowest conduction band $\left(\alpha_{n_{0}, k}\left(t_{0}\right)=\exp \left[-10\left(k / k_{\max }\right)^{2}\right]\right)$; an 8 -fs laser pulse with a central wavelength of $\lambda_{\mathrm{L}}=1.6 \mu \mathrm{m}$ and a peak field of $F_{\mathrm{L}}=0.7 \mathrm{~V} / \AA$ is sufficiently strong to accelerate the electrons out of the first Brillouin zone. (b) All electrons are initially in the valence bands; a 4-fs pulse with $\lambda_{\mathrm{L}}=800 \mathrm{~nm}$ and $F_{\mathrm{L}}=1.4 \mathrm{~V} / \AA$ excites them to the conduction bands, where they are accelerated by the laser field.

discussed in section 1.2.3. Instead, there is a transient increase of the population at the extrema of the electric field (at the zero crossings of the vector potential). Such excitations are called "virtual" [53] because they only exist as long as the external field is present. If a laser pulse is so weak that the final excitation probability can be neglected, such virtual excitations represent the distortion of bound states of the crystal - the distorted valence-band states have nonzero projections onto the field-free conduction-band Bloch functions. In the strong-field regime, no clear distinction between the "virtual" and "real" excitation can be made. Nevertheless, in both weak- and strong-field regimes, the transient increase of conduction-band population has an effect on experimentally observable quantities [53, 54].

Currently, there is no measurement technique that would reveal all the details of strong-field-driven electron dynamics like those illustrated by Fig. 1.2. However, time-resolved measurement techniques do provide indirect access to this information. Some evidence of subcycle dynamics in the strong-field excitation of electron in $\mathrm{SiO}_{2}$ was presented in [55] by measuring the polarization rotation of an elliptically polarized pulse transmitted through a thin glass plate, as well as in [56] using a noncollinear pump-probe measurement scheme. Bragg-like scattering of electrons was found to contribute to the generation of nonperturbative high-order harmonics in solid samples [7, 8]. The reversible field-induced change of absorption in the extreme ultraviolet spectral range was observed by probing the effect of an intense near-infrared field on a thin silica plate using an attosecond pulse of extreme ultra- 
violet radiation as a probe [9]. A subcycle turn-on of electric current in a dielectric and its manipulation with CEP-stabilized pulses was demonstrated by measuring the residual polarization induced by the laser light [10]. This last effect is most closely related to the topic of this chapter, so it is discussed in more detail in the following section.

\subsubsection{Ultrafast injection and control of current in dielectrics}

According to Fig. 1.2(b), an optical field that is strong enough to excite valence-band electrons of a dielectric to its conduction bands is also strong enough to significantly accelerate the created charge carriers, thus driving electric current. One of the most important findings in [10] was that such electric current can be turned on within a fraction of a half-cycle of a short intense laser pulse. This was demonstrated by irradiating a $\mathrm{SiO}_{2}$ sample placed between two gold electrodes with CEP-stabilized laser pulses (see Fig. 1.3) and measuring the current induced by the pulses in an external circuit. It was found that a short laser pulse with the electric field directed perpendicularly to the electrodes was able to leave the sample in a polarized state, implying that a certain electric charge was displaced by the pulse. By varying the CEP of the pulse, it was possible to control the amount of the displaced charge. This fact alone already suggests that the observed effect should be controlled by the electric field (rather than the envelope) of the laser pulse, but the most convincing evidence for the subcycle turn-on of electric current was provided by pump-probe measurements, where the sample was irradiated by a pair of pulses: an intense "injection pulse" polarized parallel to the electrodes and a relatively weak "drive" pulse polarized perpendicularly to them. By observing how the displaced charge depends on the delay between the two pulses, it was possible to conclude that the injection pulse makes the $\mathrm{SiO}_{2}$ sample conductive within a time interval $\lesssim 1 \mathrm{fs}$. These measurements were well reproduced by simulations: the one-dimensional tight-binding simulations in the original paper [10], a model that used a one-dimensional pseudopotential [44], and recent $a b$ initio three-dimensional simulations [57]. Nevertheless, these observations permit several interpretations, which we present in the rest of this section.

\section{Semiclassical interpretation}

The most intuitive interpretation is based on the observation that the strong-fielddriven motion of an electron wave packet in the conduction bands of a dielectric largely obeys the acceleration theorem (see Fig. 1.2(a)) — apart from interband transitions and occasional Bragg scattering, which splits and reunites electron wave packets, each wave packet moves as a classical particle with an effective mass that depends on the mean crystal momentum. This suggests that the residual polarization induced by the laser pulse may be interpreted in semiclassical terms. A rigorous 


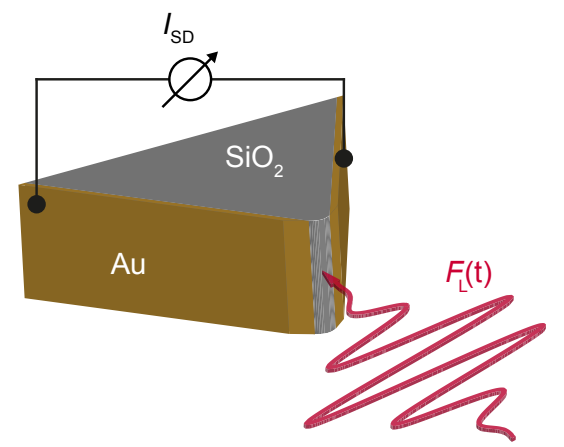

Fig. 1.3 A schematic representation of the experimental arrangement used in [10]. An intense few-cycle laser pulse induces electric current in the dielectric $\left(\mathrm{SiO}_{2}\right)$ placed between two gold electrodes. The measured signal is proportional to the net charge displaced by the laser pulse, and it is controlled by the carrier-envelope phase of the laser pulse.

approach to this interpretation consists in writing the current density averaged over a unit cell

$$
\mathbf{J}(t)=\int_{\mathrm{BZ}} \frac{\mathrm{d}^{3} k_{0}}{(2 \pi)^{3}} \mathbf{j}_{\mathbf{k}_{0}}(t)
$$

in the basis of Houston functions, where the contribution from an electron with an initial crystal momentum $\mathbf{k}_{0}$ is given by

$$
\begin{aligned}
\mathbf{j}_{\mathbf{k}_{0}}(t) & =-e \sum_{i}\left|\alpha_{i, \mathbf{k}_{0}}(t)\right|^{2} \mathbf{v}_{i}(\mathbf{k}(t)) \\
& -\frac{2 e}{m_{0}} \sum_{i, j<i} \operatorname{Re}\left\{\alpha_{i, \mathbf{k}_{0}}^{*}(t) \alpha_{j, \mathbf{k}_{0}}(t) \mathbf{p}_{i j}(\mathbf{k}(t)) \exp \left[\frac{\mathrm{i}}{\hbar} \int_{t_{0}}^{t} \mathrm{~d} t^{\prime} \Delta E_{i j}\left(\mathbf{k}\left(t^{\prime}\right)\right)\right]\right\} .
\end{aligned}
$$

Here, $\mathbf{k}(t)$ is defined by (1.23), $m_{0}$ is the electron rest mass, $\mathbf{p}_{i j}(\mathbf{k})$ are the momentum matrix elements (1.27), and $\mathbf{v}_{i}(\mathbf{k})=\nabla_{\mathbf{k}} E_{i}(\mathbf{k}) / \hbar=\mathbf{p}_{i i}(\mathbf{k}) / m_{0}$ is the group velocity in band $i$. The first sum on the right-hand side of (1.34) is responsible for the current due to the ballistic motion of charge carriers. In the semiclassical interpretation, the contribution from this term to the residual polarization (displaced charge density)

$$
\mathbf{P}\left(t_{\max }\right)=\int_{-\infty}^{t_{\max }} \mathbf{J}(t) \mathrm{d} t
$$

is assumed to be much larger than that from the second sum, which describes effects related to interband coherences.

In the tunneling regime $\left(\gamma_{\mathrm{K}} \lesssim 1\right)$, charge carriers are predominantly created at the extrema of the electric field, each of which launches an electron wave packet. For a wave packet launched at a time $t_{0}$, it is convenient to introduce a semiclassical displacement:

$$
\mathbf{s}\left(t_{0}\right)=\int_{t_{0}}^{t_{\max }} \mathbf{v}(\mathbf{k}(t)) \mathrm{d} t
$$


where the group velocity $\mathbf{v}(\mathbf{k})$ should correspond to the most probable quantum path of the wave packet in reciprocal space. As long as $\mathbf{k}(t)$ is not limited to the first Brillouin zone, this approach is most useful if the probabilities of Bragg scattering at the edges of the Brillouin zone are either negligibly small or close to $100 \%$. The contribution from each wave packet to the final polarization $\mathbf{P}\left(t_{\max }\right)$ is the product of the charge carried by the wave packet and its semiclassical displacement $\mathbf{s}\left(t_{0}\right)$.

This kind of semiclassical analysis explains some outcomes of numerical simulations [58]. In particular, it explains the observation that, for moderate laser intensities, the residual polarization scales as $P \propto F_{\mathrm{L}}^{2 N+1}$ [44], where $N=E_{\mathrm{g}} /\left(\hbar \omega_{\mathrm{L}}\right)$ is the ratio of the band gap to the photon energy. As long as the Keldysh parameter is sufficiently large to view interband excitations as the result of absorbing $N$ photons, the excitation probability scales as $p \propto I_{\mathrm{L}}^{N} \propto F_{\mathrm{L}}^{2 N}, I_{\mathrm{L}}$ being the peak laser intensity, while the semiclassical displacement is proportional to $F_{\mathrm{L}}$. Thus, the product of the charge and the displacement is proportional to $F_{\mathrm{L}}^{2 N+1}$.

\section{Interference of multiphoton pathways}

For moderate laser intensities, where perturbation theory is expected to yield at least qualitatively correct predictions, it is also possible to interpret the optically controlled electric current in terms of interference between different multiphoton excitation pathways. This interpretation is a generalization of ideas developed in the field of coherent control, where irradiating a semiconductor by two monochromatic laser beams with frequencies $\omega_{1}$ and $\omega_{2}=2 \omega_{1}$ was found to induce an electric current sensitive to the relative phase between the two beams [59]. This phase sensitivity is due to the interference between single- and two-photon absorption processes. The density $n(\mathbf{k})$ of electrons excited to the conduction band at a crystal momentum $\mathbf{k}$ is determined by the phase parameter $\Delta \varphi=2 \varphi_{\omega_{1}}-\varphi_{\omega_{2}}$. Furthermore, $n(\mathbf{k})$ is, in general, an asymmetric function of $\mathbf{k}$ because the transition amplitudes for the one- and two-photon channels have different symmetries with respect to the transformation $\mathbf{k} \rightarrow-\mathbf{k}$ [59]. Injecting currents through interfering photoexcitation pathways was investigated in theory and experiments for semiconductors [59-66] and molecular wires [67].

In the case where charge carriers are excited by a few-cycle pulse with a central frequency that is much smaller than the band gap, the interfering photoexcitation pathways are multiphoton excitation channels. It is convenient to analyze them in the velocity gauge using the basis of Bloch states, where an external homogeneous electric field only induces transitions between states with the same the crystal momentum. Due to the large bandwidth of ultrashort laser pulses, it is possible to make the same transition by absorbing different numbers of photons, as it is schematically shown in Fig. 1.4.

Within this picture, the scaling law $Q \propto F_{\mathrm{L}}^{2 N+1}$ may be interpreted as a result of interference between quantum pathways that involve absorbing $N$ and $N+1$ photons. The corresponding probability amplitudes are proportional to $F_{\mathrm{L}}^{N}$ and $F_{\mathrm{L}}^{N+1}$, respectively. When the two pathways interfere, the excitation probability, which is 


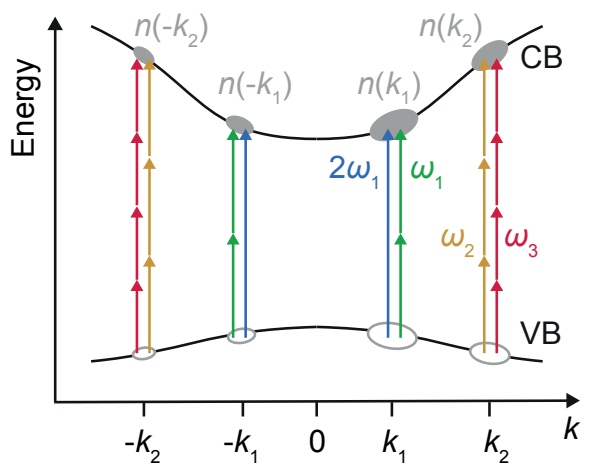

Fig. 1.4 Current injection via interference of multiphoton excitation channels. Charge carriers can be injected from a valence band (VB) into a conduction band (CB) via different multiphoton excitation pathways if the spectral bandwidth of the applied laser light is sufficiently large. For example, the excitation at crystal momenta $k_{2}$ and $-k_{2}$ may be the outcome of absorbing either three highenergy or four low-energy photons from the same ultrashort laser pulse. Quantum interference between the odd- and even-numbered contributions in each pathway determines the population in the final state. An asymmetry in the conduction-band population $(n(k) \neq n(-k))$ results in a net current density is formed inside the material.

the squared modulus of the sum of the probability amplitudes, is described by an expression that contains the product of the two amplitudes. This product, which is

proportional to $F_{\mathrm{L}}^{2 N+1}$, determines the induced electric current. Even though these arguments by no means form a rigorous proof, and these considerations are only applicable in the multiphoton regime, the interpretation in terms of interfering multiphoton channels was shown to be a plausible one, explaining not only the scaling with intensity, but also the fact that a CEP-sensitive displaced charge can only be observed if the laser pulse is sufficiently broadband [44].

\section{Adiabatic metallization}

Another interpretation of the optical-field-induced current in dielectrics accompanied the first publication of these experimental results [10]. This mechanism relies on the effect of "adiabatic metallization", predicted theoretically for dielectric nanofilms [68, 69]. It was found that a strong electric field can significantly and reversibly change the optical and electric properties of a sufficiently thin dielectric nanofilm. During the interaction with the field, the real part of the dielectric constant may even become negative, which is a property attributed to metals. These effects are best understood in the basis of instantaneous eigenstates of the lengthgauge Hamiltonian, which are the Wannier-Stark states discussed at the end of section 1.2.1. Even though these results were obtained for nanofilms, similar effects may be expected in bulk solids [70], provided that the localization length of Wannier-Stark states does not exceed a few lattice sites, which is indeed the case for field strengths $F_{\mathrm{L}} \gtrsim 1 \mathrm{~V} / \AA$. 


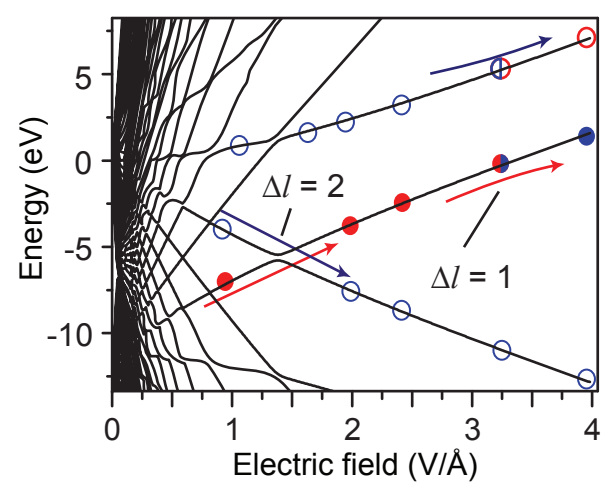

Fig. 1.5 Eigenstates of the Wannier-Stark Hamiltonian (1.8) for a 50-nm nanofilm of $\mathrm{SiO}_{2}$ in dependence of the electric field. The labels $\Delta l=1$ and $\Delta l=2$ indicate the anticrossings that correspond to Wannier-Stark states being localized one and two lattice sites apart, respectively. Closed circles represent occupied states, while open circles represent unoccupied states. The figure is adapted from Ref. [71].

Using the Wannier-Stark states as a time-dependent basis, the interaction with an intense pulse can be analyzed in terms of adiabatic and diabatic transitions at avoided crossings. This analysis $[10,71]$ shows that the strong field may cause a reversible quantum transition from an insulating state to a state with an increased conductivity. This transition occurs when the energy gaps at avoided crossings between Wannier-Stark states become sufficiently large for electrons to adiabatically pass such an anticrossing (see Fig. 1.5). The anticrossing gap takes its largest value when the relevant Wannier-Stark states are localized at neighboring lattice sites, which happens when the electric field in the medium is as strong as

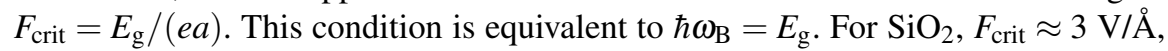
which is above the damage threshold, but even the penultimate anticrossing, which occurs at $F_{\text {crit }} \approx 1.5 \mathrm{~V} / \AA$, was found to have a sufficiently large energy gap. This interpretation of optical-field-controlled current as a result of adiabatic metallization was also supported by the observation of the optical-field-induced transient reflectivity in the strong-field regime [9].

\subsection{Summary and outlook}

The main message of this chapter is that the control over the electric field of light pulses can be transformed into the control over strong-field-driven electron dynamics, which may open attractive opportunities for both basic research and applications in ultrafast signal processing. One example of such an application is a solid-state CEP detector [72], and more advanced applications may emerge in the future. Attosecond science provides powerful tools and techniques for studying strong-field dynamics in solids, which may lead to important insights into fundamentally impor- 
tant phenomena. So far, only the first few steps have been done in this direction, and many questions remain open. Further research is necessary to verify conjectures made in the interpretation of recent measurements, and it is yet to be understood how electron-electron interaction, scattering, and dephasing phenomena affect our ability to launch and manipulate electron wave packets. One of the most important goals is to identify those extremely nonlinear effect that are largely reversible on a few-femtosecond time scale, as only such effect may serve as a basis for applications in signal processing. On the experimental side, there is a large potential for exploiting various laser sources, intense optical waveforms, and (nano) structures designed for strong-field measurements.

\section{Acknowledgments}

We acknowledge useful discussions with and help from A. Schiffrin and V. Apalkov. We thank the Munich Centre for Advanced Photonics for support. The work of M.I.S. and V.S.Y. was supported primarily by the Chemical Sciences, Biosciences and Geosciences Division (grant no. DEFG02-01ER15213) and additionally by the Materials Sciences and Engineering Division (grant no. DE-FG02-11ER46789) of the Office of the Basic Energy Sciences, Office of Science, US Department of Energy and by a MURI grant from the US Air Force Office of Scientific Research.

\section{References}

[1] M. Lenzner, J. Krüger, S. Sartania, Z. Cheng, C. Spielmann, G. Mourou, W. Kautek, F. Krausz, Phys. Rev. Lett. 80, 4076 (1998)

[2] S.S. Mao, F. Qur, S. Guizard, X. Mao, R.E. Russo, G. Petite, P. Martin, Appl. Phys. A 79(7), 1695 (2004)

[3] F. Krausz, M. Ivanov, Rev. Mod. Phys. 81, 163 (2009)

[4] E. Garmire, Opt. Express 21(25), 30532 (2013)

[5] F. Krausz, M.I. Stockman, Nature Photonics 8(3), 205 (2014)

[6] S. Ghimire, G. Ndabashimiye, A.D. DiChiara, E. Sistrunk, M.I. Stockman, P. Agostini, L.F. DiMauro, D.A. Reis, J. Phys. B 47(20), 204030 (2014)

[7] S. Ghimire, A.D. DiChiara, E. Sistrunk, P. Agostini, L.F. DiMauro, D.A. Reis, Nature Physics 7(2), 138 (2011)

[8] O. Schubert, M. Hohenleutner, F. Langer, B. Urbanek, C. Lange, U. Huttner, D. Golde, T. Meier, M. Kira, S.W. Koch, R. Huber, Nature Photonics 8(2), 119 (2014)

[9] M. Schultze, E.M. Bothschafter, A. Sommer, S. Holzner, W. Schweinberger, M. Fiess, M. Hofstetter, R. Kienberger, V. Apalkov, V.S. Yakovlev, M.I. Stockman, F. Krausz, Nature 493(7430), 75 (2013) 
[10] A. Schiffrin, T. Paasch-Colberg, N. Karpowicz, V. Apalkov, D. Gerster, S. Mühlbrandt, M. Korbman, J. Reichert, M. Schultze, S. Holzner, J.V. Barth, R. Kienberger, R. Ernstorfer, V.S. Yakovlev, M.I. Stockman, F. Krausz, Nature 493, 70 (2013)

[11] M. Wegener, Extreme Nonlinear Optics (Springer, 2005)

[12] C. Aversa, J.E. Sipe, Phys. Rev. B 52, 14636 (1995)

[13] R. Resta, Phys. Rev. Lett. 80, 1800 (1998)

[14] I. Souza, J. Íñiguez, D. Vanderbilt, Phys. Rev. B 69, 085106 (2004)

[15] K.S. Virk, J.E. Sipe, Phys. Rev. B 76, 035213 (2007)

[16] M. Springborg, B. Kirtman, Phys. Rev. B 77, 045102 (2008)

[17] W.V. Houston, Phys. Rev. 57, 184 (1940)

[18] G.H. Wannier, Phys. Rev. 117, 432 (1960)

[19] G.H. Wannier, Rev. Mod. Phys. 34, 645 (1962)

[20] P. Voisin, J. Bleuse, C. Bouche, S. Gaillard, C. Alibert, A. Regreny, Phys. Rev. Lett. 61, 1639 (1988)

[21] J. Zak, Phys. Rev. Lett. 20, 1477 (1968)

[22] G.H. Wannier, Phys. Rev. 181, 1364 (1969)

[23] J.E. Avron, J. Zak, A. Grossmann, L. Gunther, J. Math. Phys. 18(5), 918 (1977)

[24] M. Glück, A.R. Kolovsky, H.J. Korsch, Physics Reports 366(3), 103 (2002)

[25] R. Resta, J. of Phys.: Condensed Matter 12(9), R107 (2000)

[26] G. Sundaram, Q. Niu, Phys. Rev. B 59, 14915 (1999)

[27] D. Xiao, M.C. Chang, Q. Niu, Rev. Mod. Phys. 82, 1959 (2010)

[28] J. Zak, Phys. Rev. Lett. 62, 2747 (1989)

[29] R.D. King-Smith, D. Vanderbilt, Phys. Rev. B 47, 1651 (1993)

[30] M. Atala, M. Aidelsburger, J. Barreiro, D. Abanin, T. Kitagawa, Nature physics 9(12), 795 (2013)

[31] J.B. Krieger, G.J. Iafrate, Phys. Rev. B 33, 5494 (1986)

[32] D.M. Volkov, Z. Physik 94, 250 (1935)

[33] H.D. Jones, H.R. Reiss, Phys. Rev. B 16, 2466 (1977)

[34] Yu.A. Bychkov, A.M. Dykhne, Sov. Phys. JETP 58, 1734 (1970)

[35] D. Culcer, Y. Yao, Q. Niu, Phys. Rev. B 72, 085110 (2005)

[36] B.A. Foreman, J. of Phys.: Condensed Matter 12(34), R435 (2000)

[37] E.I. Blount, in Solid State Physics: Advances in Research and Applications, vol. 13, ed. by F. Seitz, D. Turnbull (Academic Press, 1962), pp. 305-373

[38] B. Gu, N.H. Kwong, R. Binder, Phys. Rev. B 87, 125301 (2013)

[39] C. Zener, Proceedings of the Royal Society of London, Series A 145(855), 523 (1934)

[40] E.O. Kane, Journal of Physics and Chemistry of Solids 12(2), 181 (1960)

[41] L.V. Keldysh, Sov. Phys. JETP 20(5), 1307 (1965)

[42] V.S. Popov, Physics-Uspekhi 47(9), 855 (2004)

[43] M. Ivanov, M. Spanner, O. Smirnova, J. Mod. Opt. 52(2-3), 165 (2005)

[44] S.Yu. Kruchinin, M. Korbman, V.S. Yakovlev, Phys. Rev. B 87, 115201 (2013)

[45] J.G. Story, D.I. Duncan, T.F. Gallagher, Phys. Rev. A 49, 3875 (1994)

[46] G.G. Paulus, F. Grasbon, H. Walther, R. Kopold, W. Becker, Phys. Rev. A 64, 021401 (2001) 
[47] R. Kopold, W. Becker, M. Kleber, G.G. Paulus, J. Phys. B 35(2), 217 (2002)

[48] M. Ivanov, O. Smirnova, Chem. Phys. 414(0), 3 (2013)

[49] H. Minasian, S. Avetisian, Phys. Rev. B 34, 963 (1986)

[50] V.A. Kovarskii, E.Yu. Perlin, Phys. Stat. Sol. B 45, 47 (1971)

[51] V.E. Gruzdev, Phys. Rev. B 75, 205106 (2007)

[52] A. Wirth, M.T. Hassan, I. Grguras, J. Gagnon, A. Moulet, T.T. Luu, S. Pabst, R. Santra, Z.A. Alahmed, A.M. Azzeer, V.S. Yakovlev, V. Pervak, F. Krausz, E. Goulielmakis, Science 334(6053), 195 (2011)

[53] E. Yablonovitch, J.P. Heritage, D.E. Aspnes, Y. Yafet, Phys. Rev. Lett. 63, 976 (1989)

[54] A.V. Kuznetsov, C.J. Stanton, Phys. Rev. B 48, 10828 (1993)

[55] M. Gertsvolf, M. Spanner, D.M. Rayner, P.B. Corkum, J. Phys. B: At. Mol. Opt. Phys. 43(13), 131002 (2010)

[56] A.V. Mitrofanov, A.J. Verhoef, E.E. Serebryannikov, J. Lumeau, L. Glebov, A.M. Zheltikov, A. Baltuška, Phys. Rev. Lett. 106, 147401 (2011)

[57] G. Wachter, C. Lemell, J. Burgdörfer, S.A. Sato, X.M. Tong, K. Yabana, Phys. Rev. Lett. 113, 087401 (2014)

[58] P. Földi, M.G. Benedict, V.S. Yakovlev, New J. of Phys. 15(6), 063019 (2013)

[59] T.M. Fortier, P.A. Roos, D.J. Jones, S.T. Cundiff, R.D.R. Bhat, J.E. Sipe, Phys. Rev. Lett. 92, 147403 (2004)

[60] G. Kurizki, M. Shapiro, P. Brumer, Phys. Rev. B 39, 3435 (1989)

[61] R. Atanasov, A. Haché, J.L.P. Hughes, H.M. van Driel, J.E. Sipe, Phys. Rev. Lett. 76, 1703 (1996)

[62] A. Haché, Y. Kostoulas, R. Atanasov, J.L.P. Hughes, J.E. Sipe, H.M. van Driel, Phys. Rev. Lett. 78, 306 (1997)

[63] J.M. Fraser, A.I. Shkrebtii, J.E. Sipe, H.M. van Driel, Physica B 272(1-4), 353 (1999)

[64] H. Zhao, E.J. Loren, A.L. Smirl, H.M. van Driel, J. Appl. Phys. 103(5), 053510 (2008)

[65] L. Costa, M. Betz, M. Spasenovic, A.D. Bristow, H.M. van Driel, Nature Physics 3(9), 632 (2007)

[66] J. Rioux, J.E. Sipe, Physica E 45(0), 1 (2012)

[67] I. Franco, M. Shapiro, P. Brumer, Phys. Rev. Lett. 99, 126802 (2007)

[68] M. Durach, A. Rusina, M.F. Kling, M.I. Stockman, Phys. Rev. Lett. 105, 086803 (2010)

[69] M. Durach, A. Rusina, M.F. Kling, M.I. Stockman, Phys. Rev. Lett. 107, $086602(2011)$

[70] V. Apalkov, M.I. Stockman, Phys. Rev. B 86, 165118 (2012)

[71] A. Schiffrin, T. Paasch-Colberg, N. Karpowicz, V. Apalkov, D. Gerster, S. Muhlbrandt, M. Korbman, J. Reichert, M. Schultze, S. Holzner, J.V. Barth, R. Kienberger, R. Ernstorfer, V.S. Yakovlev, M.I. Stockman, F. Krausz, Nature 507(7492), 386 (2014)

[72] T. Paasch-Colberg, A. Schiffrin, N. Karpowicz, S. Kruchinin, O. Sağlam, S. Keiber, O. Razskazovskaya, S. Mühlbrandt, A. Alnaser, M. Kübel, V. Apalkov, D. Gerster, J. Reichert, T. Wittmann, J.V. Barth, M.I. Stockman, 
R. Ernstorfer, V.S. Yakovlev, R. Kienberger, F. Krausz, Nature Photonics 8(3), 214 (2014) 